\title{
Error Estimates for the Clenshaw-Curtis Quadrature
}

\author{
By M. M. Chawla
}

1. Introduction. Clenshaw and Curtis [1] have proposed a quadrature scheme based on the "practical" abscissas $x_{i}=\cos (i \pi / n), \quad i=0(1) n$ and they have also discussed the estimation of error of the quadrature formula. Elliott [2] has discussed the estimation of truncation errors in the two Chebyshev series approximations for a function, one based on the practical abscissas and the other on the "classical" abscissas $x_{i}=\cos (2 i+1) \pi /(2 n+2), i=0(1) n$. Elliott also obtains asymptotic error estimates for the Lagrangian quadrature formulas based on these two sets of points. Recently, Fraser and Wilson [3] have discussed the estimation of error of the Clenshaw-Curtis quadrature and they give a simple formula for the calculation of the error in terms of the function-values.

In the present note we obtain error estimates for the Clenshaw-Curtis quadrature applied to functions analytic on the interval of integration $[-1,1]$. We also obtain error estimates for the quadrature formula based on the classical abscissas.

2. The Clenshaw-Curtis Quadrature Formula. Let $\Psi_{n}(x)$ denote the Lagrangian interpolation polynomial for $f(x)$ at the practical abscissas $x_{i}=\cos (i \pi / n)$, $i=0(1) n$, and let $\psi_{n}(x)$ denote the error of interpolation

$$
\psi_{n}(x)=f(x)-\Psi_{n}(x) .
$$

If

$$
\Psi_{n}(x)=\sum_{k=0}^{n}{ }^{\prime \prime} B_{k, n} T_{k}(x)
$$

where $T_{k}(x)=\cos (k$ arc $\cos x)$, Chebyshev polynomial of the first kind of degree $k$, and the double prime on the summation sign indicates that the first and the last terms are to be halved, then the coefficients $B_{k, n}$ are given by

$$
\begin{aligned}
B_{k, n} & =\frac{2}{n} \sum_{i=0}^{n}{ }^{\prime \prime} f\left(x_{i}\right) T_{k}\left(x_{i}\right) \\
& =\frac{2}{n} \sum_{i=0}^{n}{ }^{\prime \prime} f\left(x_{i}\right) T_{i}\left(x_{k}\right),
\end{aligned}
$$

since $T_{k}\left(x_{i}\right)=T_{i}\left(x_{k}\right)$, and $x_{i}=\cos (i \pi / n), i=0(1) n$. An elegant method for the evaluation of the coefficients $B_{k, n}$ is described by Clenshaw [4].

Let $C$ be a closed contour enclosing the interval $[-1,1]$ in its interior and let $f(z)$ be regular within $C$. Since the practical abscissas are the zeros of the polynomial $T_{n+1}(x)-T_{n-1}(x)$, the error $\psi_{n}(x)$ of the Lagrange interpolation for $f(x)$ at these abscissas can be expressed by a contour integral (Davis [5, Theorem 3.6.1]) as

Received September 29, 1967. 


$$
\psi_{n}(x)=\frac{\left[T_{n+1}(x)-T_{n-1}(x)\right]}{2 \pi i} \int_{c} \frac{f(z) d z}{(z-x)\left[T_{n+1}(z)-T_{n-1}(z)\right]}
$$

for $x \in[-1,1]$. If $n$ is even, the integration of (2) gives

$$
\int_{-1}^{1} f(x) d x \simeq \int_{-1}^{1} \Psi_{n}(x) d x=\sum_{j=0}^{n / 2} \frac{(-2) B_{2 j, n}}{4 j^{2}-1} .
$$

Substituting for $B_{2 j, n}$ from the first of the relations (3), the Clenshaw-Curtis approximate integration formula can be rewritten as

$$
\int_{-1}^{1} f(x) d x \simeq \sum_{i=0}^{n \prime \prime} \lambda_{i} f\left(x_{i}\right)
$$

where the weights $\lambda_{i}$ are given by

$$
\lambda_{i}=\frac{2}{n} \sum_{j=0}^{n / 2} \frac{\left(-\_\right) T_{2 j}\left(x_{i}\right)}{4 j^{2}-1} \text { for } i=0(1, n .
$$

The error of the Clenshaw-Curtis quadrature formula is given by

$$
E_{n}(\Psi)=\int_{-1}^{1} \psi_{n}(x) d x=\frac{1}{\pi i} \int_{C} \frac{\left[Q_{n+1}^{*}(z)-Q_{n-1}^{*}(z)\right]}{\left[T_{n+1}(z)-T_{n-1}(z)\right]} f(z) d z,
$$

where we have put

$$
Q_{n}^{*}(z)=\frac{1}{2} \int_{-1}^{1} \frac{T_{n}(x) d x}{z-x} .
$$

Equation (9) defines $Q_{n}{ }^{*}(z)$ as a single-valued analytic function in the $z$-plane with the interval $[-1,1]$ deleted.

2.1. The Quadrature Formula Based on the Classical Abscissas. Let $\Phi_{n}(x)$ denote the Lagrange interpolation polynomial for the abscissas $x_{i}=\cos (2 i+1) \pi /(2 n+2)$, $i=0(1) n$ which are the zeros of $T_{n+1}(x)$. The computation of the polynomial $\Phi_{n}(x)$ has been discussed in detail by Elliott [2].

To describe the corresponding quadrature formula, let

$$
\Phi_{n}(x)=\sum_{k=0}^{n} A_{k, n} T_{k}(x),
$$

where the prime on the summation sign indicates that the first term is to be halved. The coefficients $A_{k, n}$ are given by

$$
A_{k, n}=\frac{2}{n+1} \sum_{i=0}^{n} f\left(x_{i}\right) T_{k}\left(x_{i}\right) \text { for } i=0(1) n,
$$

where $x_{i}=\cos (2 i+1) \pi /(2 n+2)$ for $i=0(1) n$. Now, the integration of (10) gives the quadrature formula based on these abscissas as

$$
\begin{aligned}
\int_{-1}^{1} f(x) d x & \simeq \int_{-1}^{1} \Phi_{n}(x) d x \\
& \simeq \sum_{k=0}^{n} A_{k, n}\left(\int_{-1}^{1} T_{k}(x) d x\right) .
\end{aligned}
$$

But 


$$
\begin{aligned}
\int_{-1}^{1} T_{k}(x) d x & =\frac{2}{1-k^{2}} \text { if } 1-k \text { is odd } \\
& =0 \text { if } 1-k \text { is even. }
\end{aligned}
$$

Since $n$ is even, putting $k=2 m$, we get

$$
\int_{-1}^{1} f(x) d x \simeq \sum_{m=0}^{n / 2} \frac{(-2) A_{2 m, n}}{4 m^{2}-1} .
$$

Substituting for $A_{2 m, n}$ from (11), the above approximate integration formula can be put in the alternative form,

$$
\int_{-1}^{1} f(x) d x \simeq \sum_{i=0}^{n} \mu_{i} f\left(x_{i}\right)
$$

where the weights $\mu_{i}$ are given by

$$
\mu_{i}=\frac{2}{n+1} \sum_{j=0}^{n / 2 \prime} \frac{(-2) T_{2 j}\left(x_{i}\right)}{4 j^{2}-1}, \quad i=0(1) n .
$$

Let $\phi_{n}(x)=f^{\prime}(x)-\Phi_{n}(x)$ denote the error of interpolation for $f(x)$ at the classical abscissas. Then, $\phi_{n}(x)$ may be expressed in terms of a contour integral as

$$
\phi_{n}(x)=\frac{T_{n+1}(x)}{2 \pi i} \int_{C} \frac{f(z) d z}{(z-x) T_{n+1}(z)} .
$$

The error $E_{n}(\Phi)$ for the quadrature formula (14) is given by

$$
E_{n}(\Phi)=\int_{-1}^{1} \phi_{n}(x) d x=\frac{1}{\pi i} \int_{C} \frac{Q_{n+1}^{*}(z)}{T_{n+1}(z)} f(z) d z .
$$

3. A Lemma for $Q_{n}{ }^{*}(z)$. Introduce the ellipse $\varepsilon_{\rho}$ in the $z$-plane by

$$
z=\frac{1}{2}\left(\xi+\xi^{-1}\right), \quad \xi=\rho e^{i \theta}, \quad 0 \leqq \theta \leqq 2 \pi
$$

with foci at $z= \pm 1$ and whose sum of semiaxes is $\rho(\rho>1)$.

We establish the following lemma.

Lemma. For $z \in \mathcal{E}_{\rho}$,

$$
Q_{n}^{*}(z)=\xi^{-n-1} \sum_{k=-[n / 2]}^{\infty} \frac{\sigma_{n, n+2 k+1}^{*}}{\xi^{2 k}}
$$

where

$$
\sigma_{n, n+2 k+1}^{*}=2(n+2 k+1) /(2 n+2 k+1)(2 k+1),
$$

$[k]=$ greatest integer $\leqq k$.

Proof. In (9), setting $x=\cos \theta$ and transforming to the $\xi$-plane, we get

$$
Q_{n}^{*}(z)=\xi^{-1} \int_{0}^{\pi} \frac{\cos (n \theta) \sin \theta d \theta}{1-2 \cos \theta \xi^{-1}+\xi^{-2}},
$$

since $T_{n}(\cos \theta)=\cos n \theta$. Now,

$$
\frac{\sin \theta}{\xi}\left[1-2 \cos \theta \xi^{-1}+\xi^{-2}\right]^{-1}=\sum_{m=1}^{\infty} \frac{\sin m \theta}{\xi^{m}} .
$$


The last series converges uniformly and absolutely for $0 \leqq \theta \leqq \pi$ and for all $|\xi| \geqq \rho>1$. Substituting (20) in (19),

$$
Q_{n}^{*}(z)=\sum_{m=1}^{\infty} \frac{\sigma_{n m}^{*}}{\xi^{m}},
$$

where

$$
\begin{aligned}
\sigma_{n m}^{*} & =\int_{0}^{\pi} \cos n \theta \sin m \theta d \theta \\
& =\frac{2 m}{m^{2}-n^{2}} \text { if } m-n \text { is odd } \\
& =0 \text { if } m-n \text { is even } .
\end{aligned}
$$

The result follows by putting $m-n=2 k+1$ and observing that $k \geqq-[n / 2]$ for $n, m=1,2,3, \cdots$.

From the above lemma we deduce

Corollary 1. For $z \in \mathcal{E}_{\rho}$,

$$
Q_{n+1}^{*}(z)=\xi^{-n} \sum_{k=-[(n-1) / 2]}^{\infty} \frac{\sigma_{n+1, n+2 k}^{*}}{\xi^{2 k}}
$$

where

$$
\sigma_{n+1, n+2 k}^{*}=2(n+2 k) /(2 n+2 k+1)(2 k-1)
$$

and

$$
\sigma_{n+1, n+2 k}^{*} \leqq 2 n /(2 n+1) .
$$

Corollary 2. For $z \in \mathcal{E}_{\rho}$,

$$
Q_{n+1}^{*}(z)-Q_{n-1}^{*}(z)=\xi^{-n} \sum_{k=-[(n-1) / 2]}^{\infty} \frac{\lambda_{n k}^{*}}{\xi^{2 k}}
$$

where

$$
\lambda_{n k}^{*}=8 n(n+2 k) /\left[4(n+k)^{2}-1\right]\left[4 k^{2}-1\right]
$$

and

$$
\lambda_{n k}^{*} \leqq 8 n^{2} /\left(4 n^{2}-1\right) .
$$

Proof. Subtracting (18) with $n$ replaced by $n-1$ from (21),

$$
Q_{n+1}^{*}(z)-Q_{n-1}^{*}(z)=\xi^{-n} \sum_{k=-[(n-1) / 2]}^{\infty} \frac{\lambda_{n k}^{*}}{\xi^{2 k}}
$$

where

$$
\begin{aligned}
\lambda_{n k}^{*} & =\sigma_{n+1, n+2 k}^{*}-\sigma_{n-1, n+2 k}^{*} \\
& =\frac{8 n(n+2 k)}{\left[4(n+k)^{2}-1\right]\left(4 k^{2}-1\right)} .
\end{aligned}
$$

Also, 


$$
\lambda_{n k}^{*} \leqq\left|\lambda_{n, 0}\right|=\frac{8 n^{2}}{4 n^{2}-1} .
$$

4. Error Estimates. We now obtain error estimates for the Clenshaw-Curtis quadrature formula for all functions analytic on $[-1,1]$. Simultaneously, we shall obtain estimates for $E_{n}(\Phi)$.

Let $f(x)$ be analytic on $[-1,1]$. Then, for some $\rho>1, f$ can be continued analytically so as to be single valued and regular in the closure of $\varepsilon_{p}$. In (8), taking the contour to be an ellipse $\mathcal{E}_{\rho}$, we have

$$
\left|E_{n}(\Psi)\right| \leqq \frac{1}{\pi} \int_{\varepsilon_{\rho}} \frac{\left|Q_{n+1}^{*}(z)-Q_{n-1}^{*}(z)\right||f(z)||d z|}{\left|T_{n+1}(z)-T_{n-1}(z)\right|} .
$$

Now, for $n$ even, from (22) we have

$$
\begin{aligned}
\left|Q_{n+1}^{*}(z)-Q_{n-1}^{*}(z)\right| & \leqq \rho^{-n}\left(\frac{8 n^{2}}{4 n^{2}-1}\right) \sum_{k=-(n / 2)+1}^{\infty} \rho^{-2 k} \\
& \leqq\left(\frac{8 n^{2}}{4 n^{2}-1}\right)\left(\rho^{2}-1\right)^{-1}
\end{aligned}
$$

and

$$
\frac{|d z|}{\left|T_{n+1}(z)-T_{n-1}(z)\right|} \leqq \rho^{-1}\left(\rho^{n}-\rho^{-n}\right)^{-1}|d \xi| .
$$

Making use of these results, from (23) we obtain the following theorem.

THEOREM 1. Let $f(x)$ be analytic on $[-1,1]$ and be continuable analytically so as to be single valued and regular in the closure of an ellipse $\mathcal{E}_{\rho}$ with foci at $z= \pm 1$ and whose sum of semiaxes is $\rho(\rho>1)$. Then, for $n$ even,

$$
\left|E_{n}(\Psi)\right| \leqq\left(\frac{16 n^{2}}{4 n^{2}-1}\right) \frac{M(\rho)}{\left(\rho^{2}-1\right)\left(\rho^{n}-\rho^{-n}\right)}
$$

where $M(\rho)=\max _{z \in \varepsilon_{\rho}}|f(z)|$ (or equivalently on $|\xi|=\rho$ ).

4.1. We next obtain an estimate for $E_{n}(\Phi)$. From (21), we obtain for $n$ even,

$$
\begin{aligned}
\left|Q_{n+1}^{*}(z)\right| & \leqq \rho^{-n}\left(\frac{2 n}{2 n+1}\right) \sum_{k=-(n / 2)+1}^{\infty} \rho^{-2 k} \\
& \leqq\left(\frac{2 n}{2 n+1}\right)\left(\rho^{2}-1\right)^{-1} .
\end{aligned}
$$

Now, taking the contour to be an ellipse $\varepsilon_{\rho}$ in (17), we have

$$
\left|E_{n}(\Phi)\right| \leqq \frac{1}{\pi} \int_{\varepsilon_{\rho}} \frac{\left|Q_{n+1}^{*}(z)\right||f(z)||d z|}{\left|T_{n+1}(z)\right|} .
$$

Employing (25), we obtain the following theorem from (26).

Theorem 2. Let $f(x)$ satisfy the regularity conditions of Theorem 1. Then, for $n$ even,

$$
\left|E_{n}(\Phi)\right| \leqq\left(\frac{4 n}{2 n+1}\right) \frac{\left(\rho+\rho^{-1}\right)}{\left(\rho^{2}-1\right)} \frac{M(\rho)}{\left(\rho^{n+1}-\rho^{-n-1}\right)} .
$$


Remarks. (i) From (24) and (27) it would appear that the estimate for $E_{n}(\Phi)$ is nearly half of that for $E_{n}(\Psi)$ for large $n$ and $\rho \gg 1$. For small values of $n$ and $\rho$ near 1 , the estimate (27) is still less than the estimate (24).

(ii) The estimates (24) and (27) obtained for the ellipse will be reasonably reliable for large $\rho$, while these estimates are poor if $\rho$ is near 1 .

(iii) For fixed $n$ and varying $\rho$, a "least conservative" upper bound can be established for these estimates for some $\rho^{*}\left(1<\rho^{*} \leqq \rho\right)$. However, observe that if $f(z)$ is entire, $\rho^{*}$ will be a value of $\rho$ for which the right side of (24) or (27) is a minimum.

5. Example. We illustrate the error estimate (24) for the Clenshaw-Curtis quadrature for the function $f(x)=1 /(x+4)$, and compare the estimates obtained with those given by Fraser and Wilson [3].

We select $\rho=7$ for which $f(z)=1 /(z+4)$ is regular within the closed ellipse $\mathcal{E}_{7}$. Now, on $\mathcal{E}_{\rho}$,

$$
|f(z)| \leqq M(\rho)=\frac{2 \rho}{\left(4+(15)^{1 / 2}-\rho\right)\left(\rho-\left(4-(15)^{1 / 2}\right)\right)}
$$

Thus, $M(7) \doteq 2.3333333347$.

The estimate (24) for the error of the Clenshaw-Curtis quadrature applied to this function is given by

$$
\left|E_{n}(\Psi)\right| \leqq\left(\frac{n^{2}}{4 n^{2}-1}\right) \frac{M(7)}{\left(7^{n}-7^{-n}\right)} .
$$

The exact value of $\int_{-1}^{1} d x /(4+x) \doteq 0.51082562$.

TABLE I

\begin{tabular}{lllllll}
\hline$n$ & \multicolumn{2}{c}{ estimated error } & \multicolumn{2}{c}{ actual error } & \multicolumn{3}{c}{$\begin{array}{c}\text { estimates of } \\
\text { Fraser-Wilson }\end{array}$} \\
\hline 2 & 0.0042 & 3456 & 0.0002 & 8549 & 0.0166 & 6667 \\
4 & 0.0000 & 8230 & 0.0000 & 0125 & 0.0002 & 6882 \\
8 & 0.0000 & 00027 & 0.0000 & 0000 & 0.0000 & 0007 \\
\hline
\end{tabular}

The error estimated by (28) is compared in Table I with the actual error and the estimates given by Fraser and Wilson [3].

Department of Mathematics

Indian Institute of Technology

Hauz Khas, New Delhi-29

India

1. C. W. Clenshaw \& A. R. Cur'tis, "A method for numerical integration on an automatic computer," Numer. Math., v. 2, 1960, pp. 197-205. MR $22 \# 8659$.

2. D. ELLIOTT, "Truncation errors in two Chebyshev series approximations," Math. Comp., v. 19, 1965, pp. 234-248. MR 31 \#5313.

3. W. FRASER \& M. W. WILSON, "Remarks on the Clenshaw-Curtis quadrature scheme," SIAM Rev., v. 8, 1966, pp. 322-327. MR 34 \#3784.

4. C. W. Clenshaw, Chebyshev Series for Mathematical Functions, National Physical Laboratory Mathematical Tables, Vol. 5, Department of Scientific and Industrial Research, H. M. S. O., London, 1962. MR $26 \# 362$.

5. P. J. DAvis, Interpolation and Approximation, Blaisdell, New York, 1963, pp. 67-68, 311312. MR $28 \# 393$. 